\title{
Suture pattern does not influence outcomes of endoscopic sleeve gastroplasty in obese patients
}

\section{(ㄷ)(요 $\odot$}

\author{
Authors
}

E. Espinet-Coll1,2, J. Nebreda-Durán², M. Galvao-Neto³ , C. Bautista-Altamirano4 ${ }^{4}$, P. Diaz-Galán', J. A. Gómez-Valero¹, C. Vila-Lolo', M. A. Guirola-Puche ${ }^{2}$, A. Fernández-Huélamo² ${ }^{2}$ D. Bargalló-Carulla², A. Juan-Creix Comamala1 ture pattern used. submitted 24.4.2020

accepted after revision 12.6 .2020

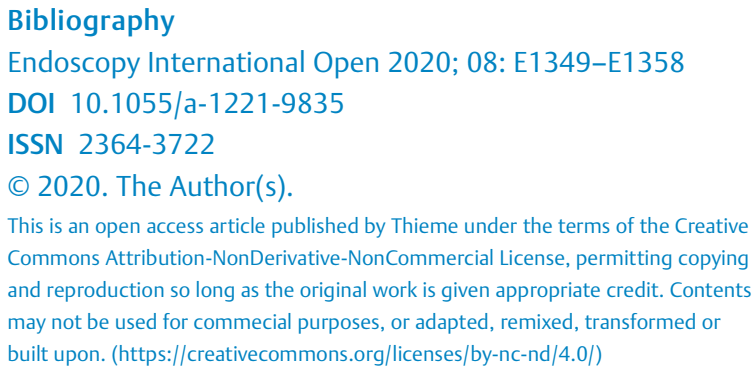
Commons Attribution-NonDerivative-NonCommercial License, permitting copying and reproduction so long as the original work is given appropriate credit. Contents may not be used for commecial purposes, or adapted, remixed, transformed or built upon. (https://creativecommons.org/licenses/by-nc-nd/4.0/)

Corresponding author

Eduard Espinet-Coll , Digestive and Bariatric Endoscopy Unit, Dexeus University Hospital, Gran Vía Carlos III, 71-75, 08028 Barcelona, Spain

Fax: +93 4177589

eespinet@hotmail.com

\section{ABSTRACT}

Background and study aims ESG is an effective and safe medium-term procedure for obesity treatment. A variety of suture patterns have been reported. We aimed to compare whether there are differences in efficacy depending on su-

Patients and methods Retrospective and comparative review of 5 years of prospectively collected data, including consecutive obese patients undergoing ESG at two collaborative centers. Primary outcomes included weight loss (mainly \% total body weight loss [TBWL] and \% exces weight loss [EWL]) at 12 months and safety profile. We compared them according to three suture patterns (transverse bilinear [TBp], longitudinal [LP] and transverse monolinear [TMp]), and number of sutures ( $4-7)$ and stitches $(<25,25$ to 30 and >30) applied. Evolution of major obesity-associated morbidities (hypertension, dyslipidemia, Type 2 diabetes mellitus (T2DM), sleep obstructive apnea syndrome, and arthropathy) were also described.

Results 88 patients (mean age $46.1 \pm 12.3$ years, $69.3 \% \mathrm{fe}-$ male) underwent ESG. Mean body mass index (BMI) at baseline was $39.40 \pm 4.69 \mathrm{~kg} / \mathrm{m}^{2}$. At 1 year, \%TBWL was $17.36 \pm$ $6.09 \%$ (\%EWL $46.41 \pm 20.6 \%$ ) with TBWL $>10 \%$ in $95.5 \%$ of patients (EWL $>25 \%$ in $94.3 \%$ of patients). According to pattern, there were no differences in \%TBWL but there were in \%EWL $(43.7 \pm 20.4 \%, 59.8 \pm 18.9 \%$ and $45.4 \pm 14.9 \%$ in TBp, Lp and TMp patterns, respectively) $(P=0.034)$. No differences were found related to number of sutures (mean 5.2 $\pm 0.73, r=4-7$ ) or stitches (mean 27.4 $\pm 6.50, r=$ $18-50)$ applied. Forty-three of $72(59.7 \%)$ major comorbidities were resolved. No serious adverse events were observed with any pattern.

Conclusions ESG is an effective procedure at 12-month follow-up for weight loss and comorbidity resolution. All three analyzed patterns are safe and effective without differences in \%TBWL, but there was a slight increase in \%EWL in Lp, regardless of the number of sutures or stitches applied.

\section{Introduction}

Gastric restrictive bariatric endoscopy techniques have gained standing in the arsenal of weight loss therapies because of their minimally invasive nature, reversibility, and applicability in patients otherwise ineligible for bariatric surgery [1]. To date, different models of intragastric balloons and several methods of stapling and suturing have been reported.
Endoscopic sleeve gastroplasty (ESG) is an incisionless, minimally invasive, full-thickness bariatric endoscopic suturing system [2,3]. Multiple case series and several meta-analyses have proved its technical feasibility, safety, and effectiveness in medium-term results [4-7]. A variety of ESG suture patterns have been reported, with no single standardized or universal pattern. Most of these studies use a suture pattern typically 
from the hospital or clinical trial where the procedure was performed ( $\triangleright$ Fig. 1). To date, no study has specifically compared ESG results based on the suture pattern used.

We report, to our knowledge, the first study on ESG technique comparing efficacy (weight loss results and major obesity-associated metabolic diseases evolution) and safety (major adverse events [AEs]) in three different suture patterns and distribution of suture placement, and related to number of sutures and total number of stitches applied.

\section{Patients and methods}

A descriptive, comparative, retrospective review study with prospective data collection was designed. Ninety-four consecutive patients underwent ESG between March 2014 and February 2019 at two hospitals in Barcelona, Spain (Dexeus University Hospital and Diagonal Clinic). All overall data and results were expressed at 12-month follow-up. Six patients were dismissed for not complying with the 12-month follow-up: family problems and geographical distance were causes. It was confirmed that none of them had complications. A total of $88 \mathrm{pa}$ tients were included.

\section{Study population}

Inclusion criteria were: 1) overweight grade II or obesity grade I-II patients (body mass index [BMI] $27-39.9 \mathrm{~kg} / \mathrm{m}^{2}$ ); 2) morbidly obese patients (BMI>40 kg/m²) who refused surgery; 3 ) age 18 to 75 years; 4) ability to understand weight lost mechanisms after endoscopic treatment; 5) acceptance and understanding of treatment objectives; and 6) commitment to adherence to follow-up for at least 1 year. The procedure was contraindicated in presence of gastric inflammatory pathology, coagulopathies or major psychiatric disorders, all as subsequently defined in Spanish Consensus Document on Bariatric Endoscopy [8].

\section{Technical description}

All procedures were performed by two endoscopists with similar training (EEC and JND), in the same two city hospitals. Gastroplasty was performed using an endoscopic suturing system (OverStitch, Apollo Endosurgery Inc., Austin, Texas, USA) inserted into the end of a double-channel therapeutic upper endoscope (GIF-2T140 and GIF-2T160, Olympus Medical Systems Corp, Tokyo, Japan). The process was carried out under general anesthesia and endotracheal intubation controlled by an anesthetist, regardless of supine decubitus or left lateral position. A 25-cm, 17-mm ID-specific esophageal overtube was used. Insufflation with $\mathrm{CO}_{2}$ and antibiotic (cefotaxime or amoxicillinclavulanic, $2 \mathrm{~g}$ intravenous [IV], single dose) and antacid (pantoprazole, $40 \mathrm{mg} \mathrm{IV}$, in bolus) pre-procedure prophylaxis were administered. After the procedure, all patients were admitted overnight for observation.

Sutures were placed from distal to proximal, starting at incisura angularis and ending up in fundus, creating a gastric sleeve. Antrum and gastric lesser curvature remained free of sutures in all cases. No reinforcing sutures were used in any case. Finally, gastroscopy was performed to confirm the final gastric tubular configuration and to rule out any potential immediate complication.

\section{Suture patterns}

Three different suture patterns were applied ( $>$ Fig. 1).

From March 2014 to December 2015, a transverse bilinear pattern (TBP) was used ( Fig. 1a, > Fig. 2a, > Fig. 2b). Triangu-

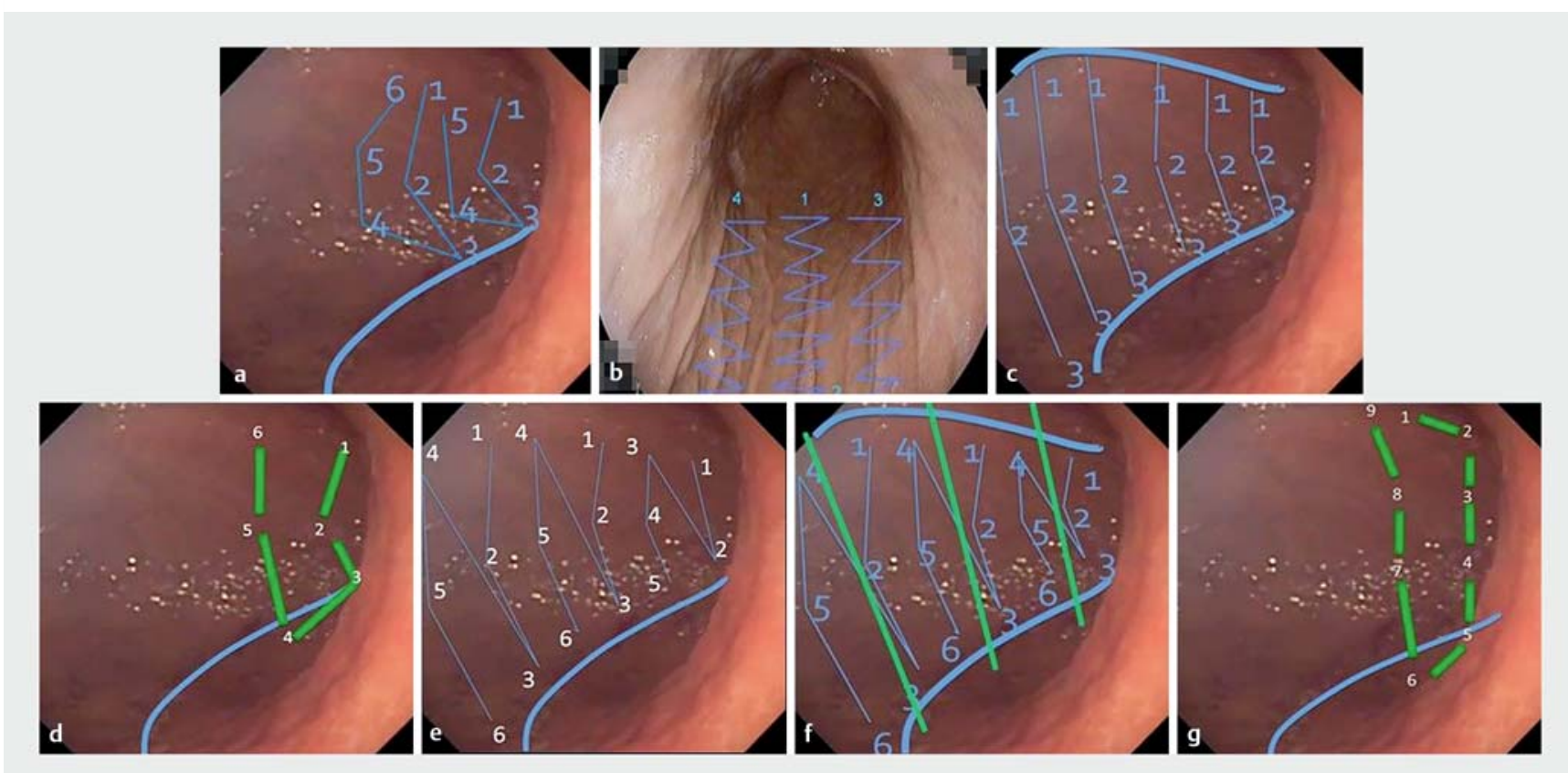

- Fig. 1 Different types of endoscopic suture patterns used in ESG-Apollo. Images a (TBp), b (LP) and c (TMp) correspond to the patterns used in our study. 


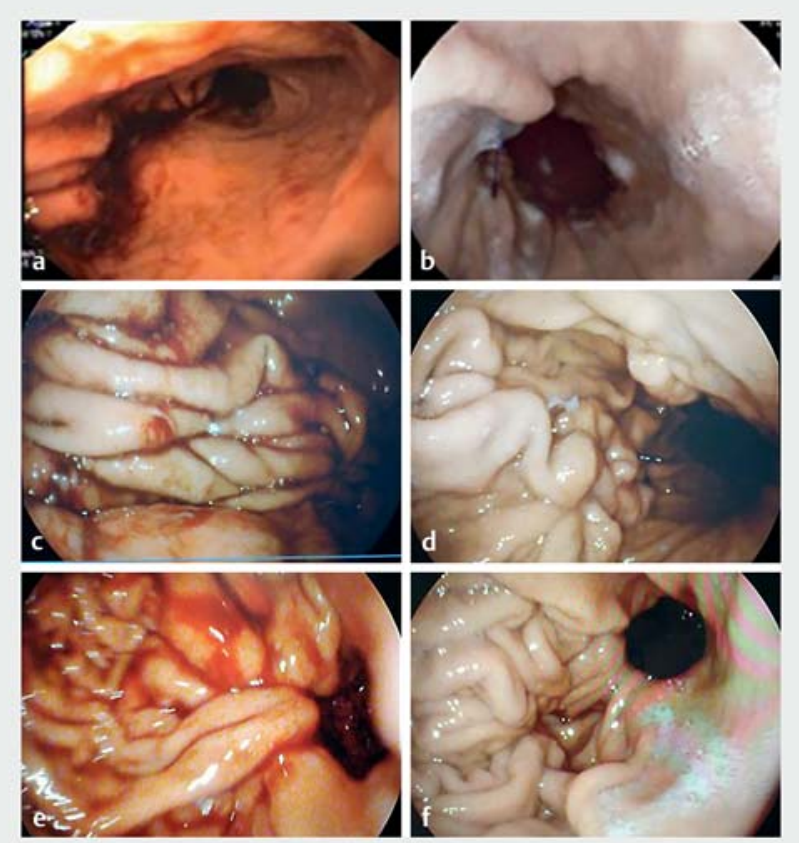

- Fig. 2 a, b TBp post-procedure immediately and 12-month follow-up. c, $\mathbf{d}$ Lp post-procedure immediately and 12-month followup. e,f TMp post-procedure immediately and 12-month follow-up.

lar suturing pattern was performed starting at anterior wall, followed by greater curvature and posterior wall. Then the pattern was repeated, in the opposite direction to 1 to $2 \mathrm{~cm}$ proximal, with the same suture. Forty-one patients were in this group.

From January 2016 to December 2017 a longitudinal pattern in "z" (Lp) was applied ( $\triangleright$ Fig. 1b, > Fig. 2c, $>$ Fig. 2d), in longitudinal rows of parallel sutures along gastric body. First the greater curvature was sutured, then the anterior wall and finally the posterior wall of the gastric body. Twenty-three patients were included in this group.

From January 2018 to February 2019 a transverse monolinear pattern (TMp) was used (> Fig. 1c, $>$ Fig. 2e, $>$ Fig. 2f). It consisted of a linear suturing pattern, starting at the anterior wall, followed by the greater curvature and finally ending up on the posterior wall, where the suture was cinched and finalized. Twenty-four patients were in this group.

The number of sutures applied in absolute values and number of stitches (bites) made were counted in each procedure. Number of stitches were arbitrarily divided into three categories: $<25,25$ to 30 and $>30$ stitches (according to $33-66-100$ percentiles of all evaluated procedures).

\section{Follow-up}

A liquid diet was started at 4 to 6 hours and for 2 weeks, followed by soft intake ( 2 weeks) and advanced to hypocaloric normal diet from the first month after the procedure. Oral analgesic and antiemetic regimen were maintained for 3 days and proton pump inhibitors for 2 months. Follow-up in person or by telephone were done at Weeks 1, 2, and 4, and subsequently once a month by Nutrition-Dietetic, and quarterly by Psycholo- gy support (12 months at least). No anorexygen treatment was used and gastroplasty, diet, and changes in lifestyle were the only factors that, a priori, influenced results.

\section{Definitions and outcomes}

The primary outcome was efficacy data at 12 months. Percentage of total body weight loss (\%TBWL) and excess weight loss (\%EWL) were prioritized as comparative anthropometric parameters, although we also collected weight loss (WL, $\mathrm{kg}$ ) and BMI $\left(\mathrm{kg} / \mathrm{m}^{2}\right)$ variations. Clinical success was defined as TBWL> $10 \%$ and $\mathrm{EWL}>25 \%$, both in $>75 \%$ of patients, at 1 -year control, as the Spanish Consensus Document on Bariatric Endoscopy [8] establishes.

Secondary outcomes included impact of ESG on five obesityassociated major comorbidities according to the Spanish Society for Obesity Surgery (SECO). They were defined according to metabolic syndrome criteria described in Circulation [9]: 1) dyslipidemia (DLP): triglycerides $>150 \mathrm{mg} / \mathrm{dL}$ and/or LDL $>170 \mathrm{mg} /$ $\mathrm{dL}$ and/or $\mathrm{HDL}<50 \mathrm{mg} / \mathrm{dL}$ in males or $<40 \mathrm{mg} / \mathrm{dL}$ in females, or into hypolipidemic pharmacological treatment; 2) arterial hypertension $(\mathrm{AHT})>130 / 85 \mathrm{mmHg}$, or in antihypertensive medication; 3) Type 2 diabetes mellitus (T2DM): fasting glucose $>100 \mathrm{mg} / \mathrm{dL}, \mathrm{HbA} 1 \mathrm{c}>6.5 \%$ or previous diagnosis of DM or in treatment with antidiabetic drugs; 4) sleep obstructive apnea syndrome (SOAS); and 5) severe arthropathy in load joints, subjectively evaluated according to specific anamnesis to patients and their relatives. Resolution of a comorbidity was defined when parameters were in normal range, drugs could be discontinued, and/or clinical symptoms disappeared.

\section{Safety}

Data on tolerance, safety, and major AEs were collected at each control, according to updated validated Clavien-Dindo surgical classification [10]. For any AE, its resolution, whether medical, endoscopic or surgical, was documented. Accommodative symptoms, from the first week, were not taken into account.

\section{Statistical analysis}

Descriptive analysis of continuous and non-continuous quantitative variables and qualitative were calculated and expressed using mean and standard deviation (mean $\pm S D$ ), mean and interquartile range (mean $\pm I Q R$ ) and frequencies and percentages, respectively. Normal distribution of quantitative continuous variables was evaluated via Shapiro-Wilk test, with a significance level of $P=0.01$. ANOVA test or Kruskal-Wallis test were used for suture pattern, number of sutures, and stitches comparison. In addition, Chi-square test was used for the comparison between categorical variables.

$P<0.05$ was considered statistically significant. All analyses were performed using RStudio Team (2019) program (RStudio: Integrated Development for RStudio, Inc., Boston, Massachusetts, United States; http://www.rstudio.com/).

\section{Ethics aspects}

Dexeus University Hospital Ethical Committee in Barcelona, Spain, approved this study. A specific informed consent for endoscopic treatment was reviewed and signed. Surveillance 
was partially funded by a grant from Spanish Society for Gastrointestinal Endoscopy (SEED) in 2016 and financial support granted by Dexeus University Hospital in 2018 for statistical study.

\section{Results}

Eighty-eight patients (27 male, 61 female) with mean age $46.1 \pm$ 12.3 years (range $18-74$ ) were enrolled ( $>$ Table 1 ). All patients had at least 12 months of follow-up. Baseline mean weight was $110.71 \pm 17.9 \mathrm{~kg}$ (range $72-160 \mathrm{~kg}$ ), equivalent to mean BMI of $39.40 \pm 4.69 \mathrm{~kg} / \mathrm{m}^{2}$ (range $29.6-52.0 \mathrm{~kg} / \mathrm{m}^{2}$ ) and 34 patients (38.6\%) had at least one major obesity-related comorbidity, for a total of 72 major comorbidities among all patients.

At 1 year, mean \%TBWL and \%EWL were $17.36 \pm 6.09 \%$ (range $8.6 \%-33.0 \%$ ) and $46.41 \pm 20.6 \%$ (range $16.0 \%-94.2 \%$ ) with TBWL $>10 \%$ and $\mathrm{EWL}>25 \%$ in $95.5 \%$ and $94.3 \%$ patients, respectively. Sixteen of 34 patients $(47.1 \%)$ with some initial comorbidity had none of them at the end of the study. Forty-three of 72 initial comorbidities were resolved (59.7\% resolution) and improvement was seen in all comorbidities analyzed ( $\triangleright$ Table 1 ).

Each of the three suture patterns used was effective in weight loss ( $\triangleright$ Table 2, $>$ Fig. 3a, $\triangleright$ Fig. 3b). TBWL $>10 \%$ and EWL $>25 \%$ were achieved in more than $91 \%$ of patients with each pattern. We obtained \%EWL of $43.7 \pm 20.4 \%, 59.8 \pm 18.9 \%$ and $45.4 \pm 14.9 \%$ with TBp, Lp and TMp patterns respectively, statistically significant $(P=0.034)$, but with differences in baseline BMI $(P=0.008)$. These differences were not significant for $\%$ TBWL $(P=0.093)$ or WL $(\mathrm{kg})(P=0.096)$. A higher number of sutures were applied with the TBp pattern and a higher number of stitches with the Lp pattern $(P<0.001)$.

An average of $5.2 \pm 0.73$ sutures/patient were used (range 4-7) ( $\triangleright$ Table 3). At 12 months, treatment was effective regardless of number of sutures applied: TBWL $>10 \%$ and EWL > $25 \%$ were achieved in more than $92 \%$ of patients with each one of suture groups. There were no significant differences in \%TBWL $(P=0.949)$ or \%EWL $(P=0.296)$ depending on number of sutures applied ( $\vee$ Fig.3c). A direct relationship was observed between number of sutures and number of stitches applied $(P=0.052)$.

An average of $27.4 \pm 6.50$ stitches/patient were performed (range 18-50) ( Table 4). Treatment was effective regardless of number of stitches used: TBWL $>10 \%$ and EWL $>25 \%$ were achieved in more than $93 \%$ of patients with each stitches group. There were no differences in \%TBWL $(P=0.911)$ or \% EWL $(P=0.223)$ between patients who received $<25,25$ to 30 , or $>30$ stitches ( $>$ Fig. $\mathbf{3 d}$ ). Patients receiving more than 25 stitches had more sutures $(P=0.026)$.

No severe AEs or major complications were observed in any of patterns ( Table 1). As minor incidences, dysphagia and abdominal pain symptoms required prolonging hospital admission by 24 hours in two patients (2.3\%), both with TBp suture pattern and managed with medications. Symptoms AEs were self-limited and did not require any further exploration. All patients were free of analgesic and antiemetic medication on Day 7 and all remained in grade I of Clavien-Dindo classification. There were no cases of bleeding, hospital re-entry, surgical requirement, or mortality.
- Table 1 General demographic, efficacy and safety data.

\begin{tabular}{|c|c|c|}
\hline Data & $\begin{array}{l}\text { Baseline } \\
(n=88)\end{array}$ & $\begin{array}{l}\text { Final (12-months) } \\
(\mathrm{n}=88)\end{array}$ \\
\hline Age, mean (SD), years & $46.1(12.3)$ & \\
\hline \multicolumn{2}{|l|}{ Gender } & \\
\hline Male, n (\%) & $27(30.7 \%)$ & \\
\hline Female, n (\%) & $61(69.3 \%)$ & \\
\hline Weight, mean (SD), kg & $110.71(17.9)$ & $91.50(16.9)$ \\
\hline WL, mean (SD), kg & & $19.21(6.34)$ \\
\hline BMI, mean (SD), kg/m² & $39.40(4.69)$ & $33.30(5.21)$ \\
\hline BMIL, mean (SD), $\mathrm{kg} / \mathrm{m}^{2}$ & & $6.10(0.52)$ \\
\hline \%TBWL, mean (SD), \% & & $17.36(6.09 \%)$ \\
\hline TBWL> $10 \%, n(\%)$ & & $84 / 88$ (95.5\%) \\
\hline \%EWL, mean (SD), \% & & $46.41(20.6 \%)$ \\
\hline$E W L>25 \%, n(\%)$ & & $83 / 88$ (94.3\%) \\
\hline \multicolumn{3}{|l|}{ Comorbidities } \\
\hline $\begin{array}{l}\text { Affected patients, } \\
\mathrm{n}(\%)\end{array}$ & $34(38.6 \%)$ & $\begin{array}{l}18(20.4 \%)(47.1 \% \\
\text { reduction) }\end{array}$ \\
\hline - AHT, n (\%) & $16(18.2 \%)$ & $\begin{array}{l}8(9.1 \%)(50 \% \\
\text { reduction) }\end{array}$ \\
\hline - DLP, n (\%) & $24(27.3 \%)$ & $\begin{array}{l}10(11.4 \%)(58 \% \\
\text { reduction) }\end{array}$ \\
\hline - T2DM, n (\%) & $11(12.5 \%)$ & $\begin{array}{l}6(6.8 \%)(45.5 \% \\
\text { reduction) }\end{array}$ \\
\hline - SOAS, n (\%) & $8(9.1 \%)$ & $\begin{array}{l}3(3.4 \%)(62.5 \% \\
\text { reduction) }\end{array}$ \\
\hline - Arthropathy, n (\%) & $13(14.8 \%)$ & $\begin{array}{l}2(2.3 \%)(84.5 \% \\
\text { reduction) }\end{array}$ \\
\hline - Total, n & 72 & 29 ( $59.7 \%$ reduction) \\
\hline \multicolumn{3}{|l|}{ Adverse events } \\
\hline - Major, n (\%) & & $0(0.0 \%)$ \\
\hline - Minor, n (\%) & & $2(2.3 \%)^{*}$ \\
\hline $\begin{array}{l}\text { - Clavien-Dindo: } \\
\text { Grade I, n (\%) }\end{array}$ & & $88(100 \%)$ \\
\hline
\end{tabular}

WL, weight loss; BMI, body mass index; BMIL, body mass index loss; TBWL, total body weight loss; TBWL $>10 \%$, number of patients with TBWL $>10 \%$; EWL, excess weight loss; EWL $>25 \%$, number of patients with $E W L>25 \%$; AHT, Arterial hypertension; DLP, Dyslipidemia; T2DM, Type 2 diabetes mellitus; SOAS, sleep obstructive apnea syndrome.

* Pain/dysphagia self-limiting in 48 hours.

\section{Discussion}

The goal of ESG is to reduce gastric volume and compliance, delay gastric emptying, induce early satiation and, possibly, alter gut and metabolic hormones [11]. These are achieved by creating a gastric sleeve, made via endoluminally placed full-thickness (serosa-to-serosa) sutures, giving durability to the procedure. 
- Table 2 baseline and 12-month anthropometric data according to suture pattern

\begin{tabular}{|c|c|c|c|c|}
\hline \multirow[t]{2}{*}{ Suture pattern } & TBp & Lp & TMp & P overall \\
\hline & $(n=41)$ & $(n=23)$ & $(n=24)$ & \\
\hline Gender: & & & & 0.313 \\
\hline - Male, n (\%) & $10(38.5 \%)$ & $2(14.3 \%)$ & $5(33.3 \%)$ & \\
\hline - Female, n (\%) & $16(61.5 \%)$ & $12(85.7 \%)$ & $10(66.7 \%)$ & \\
\hline Age, mean (SD), years & $44.6(13.4)$ & $50.8(11.3)$ & $44.2(10.5)$ & 0.253 \\
\hline $\mathrm{W}_{\mathrm{o}}$, mean $(\mathrm{SD}), \mathrm{kg}$ & $115(17.4)$ & $103(20.5)$ & $110(14.8)$ & 0.149 \\
\hline $\mathrm{BMI}_{0}$, mean $(\mathrm{SD}) \mathrm{kg} / \mathrm{m}^{2}$ & $41.4(5.09)$ & $37.0(3.88)$ & $38.2(3.19)$ & 0.008 \\
\hline No. sutures, mean (SD) & $5.77(0.71)$ & $4.93(0.62)$ & $4.73(0.80)$ & $<0.001$ \\
\hline No. sutures: & & & & 0.001 \\
\hline - $4, \mathrm{n}(\%)$ & $0(0.00 \%)$ & $5(21.7 \%)$ & $11(45.8 \%)$ & \\
\hline - $5, \mathrm{n}(\%)$ & $15(36.6 \%)$ & $15(65.2 \%)$ & $8(33.3 \%)$ & \\
\hline - $6, n(\%)$ & $19(46.3 \%)$ & $3(13.1 \%)$ & $5(20.9 \%)$ & \\
\hline - 7, n (\%) & $7(17.1 \%)$ & $0(0.00 \%)$ & $0(0.00 \%)$ & \\
\hline No. stitches, mean (SD) & $26.4(4.92)$ & $33.1(7.85)$ & $23.7(3.62)$ & $<0.001$ \\
\hline No. stitches & & & & 0.001 \\
\hline - $<25, \mathrm{n}(\%)$ & $17(41.5 \%)$ & $3(13.1 \%)$ & $17(70.8 \%)$ & \\
\hline - $25-30, n(\%)$ & $18(43.9 \%)$ & $5(21.7 \%)$ & $7(29.2 \%)$ & \\
\hline - >30, n (\%) & $6(14.6 \%)$ & $15(65.2 \%)$ & $0(0.00 \%)$ & \\
\hline$W_{12}$, mean $(S D), k g$ & $94.1(17.6)$ & $84.0(18.3)$ & $93.9(12.9)$ & 0.163 \\
\hline $\mathrm{BMI}_{12}$, mean $(\mathrm{SD}), \mathrm{kg} / \mathrm{m}^{2}$ & $35.0(5.84)$ & $30.7(4.00)$ & $33.1(2.70)$ & 0.095 \\
\hline $\mathrm{WL}_{12}$, mean $(\mathrm{SD}), \mathrm{kg}$ & $20.8(6.45)$ & $19.6(7.28)$ & $16.4(4.28)$ & 0.096 \\
\hline$\% \mathrm{EWL}_{12}$, mean (SD), \% & $43.7(20.4)$ & $59.8(18.9)$ & $45.4(14.9)$ & 0.034 \\
\hline $\mathrm{EWL}_{12}$ : & & & & 0.398 \\
\hline . $<25 \%, \mathrm{n}(\%)$ & $3(7.3 \%)$ & $0(0.00 \%)$ & $2(8.3 \%)$ & \\
\hline . $>25 \%, n(\%)$ & $38(92.7 \%)$ & $23(100 \%)$ & $22(91.7 \%)$ & \\
\hline$\% \mathrm{TBWL}_{12}$, Mean (SD), \% & $18.3(6.03)$ & $19.1(6.37)$ & $14.9(3.23)$ & 0.093 \\
\hline TBWL $_{12}:$ & & & & 1.000 \\
\hline - < $10 \%, \mathrm{n}(\%)$ & $2(4.9 \%)$ & $0(0.00 \%)$ & $2(8.3 \%)$ & \\
\hline - > $10 \%, \mathrm{n}(\%)$ & $39(95.1 \%)$ & $23(100 \%)$ & $22(91.7 \%)$ & \\
\hline
\end{tabular}

In 2013, Abu Dayyeh et al. [3] first documented the feasibility and safety of the OverStitch endoscopic suturing system (Apollo Endosurgery, Austin, Texas, United States) in four patients. Initial cases were performed using running stitches with 6 to 12 tissue areas and in a triangular fashion. A variety of stitch patterns and sequences were studied, iteratively optimized and reported, typically by the hospital or in a clinical trial in which the procedure was performed ( $\triangleright$ Fig. $\mathbf{1}$ ). Dozens of studies and several meta-analyses [4-6] have subsequently been published. This is, to our knowledge, the first ESG study comparing efficacy and safety results among three different suture patterns and number of sutures and stitches used for ESG technique.

Pooled results of \%TBWL from three comprehensive systematic review and meta-analyses [4-6], at 12-month followup were $16.09 \%$ to $16.5 \%$ (\%EWL $59 \%-61 \%$ ). Our overall results are similar, resulting in a slightly higher average \%TBWL but with somewhat lower mean \%EWL, likely due to highest baseline mean BMI in our patient cohort. In any case, we obtained 


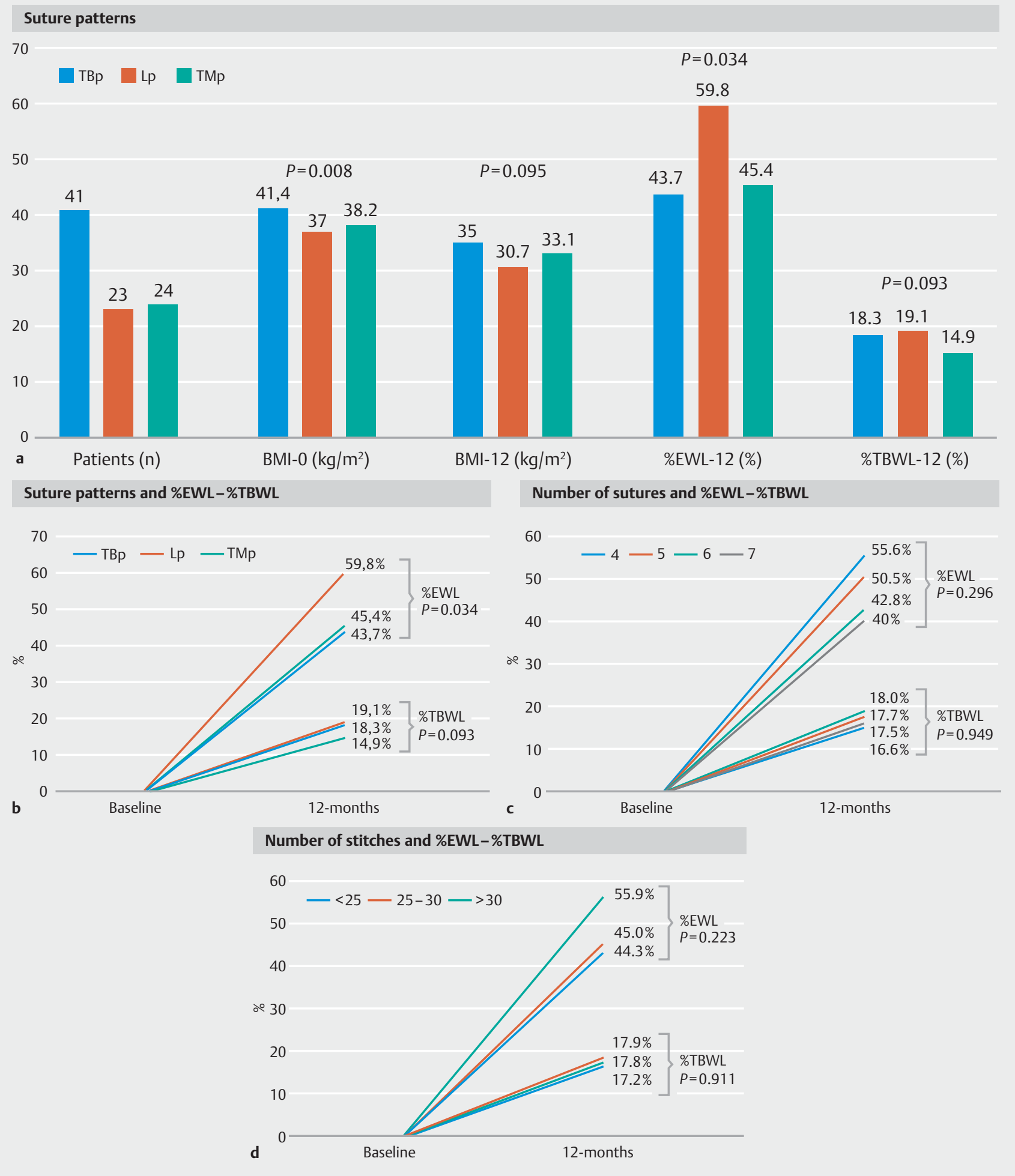

- Fig. 3 ESG suture PATTERN, SUTURES and STITCHES impact on \%EWL and \%TBWL at 12-month follow-up.

TBWL $>10 \%$ and $\%$ EWL $>25 \%$ in more than $75 \%$ of patients, confirming efficacy of our treatment, although with limited followup to 12 months. Long-term results have not been reported but a study [12] notes that 6 -month weight-loss may be a 2 -year efficacy predictor and a recent presentation (unpublished data)
[13] showed \%TBWL of $14.5 \%$ at 5 years, with maximum weight loss being at 24 months.

To date, most studies have used a transversal bilinear suture pattern variant. Using it, in the largest prospective study published to date [14], the mean \%TBWL attained at 12 months 
- Table 3 Baseline and 12-month anthropometric data according to number of sutures.

\begin{tabular}{|c|c|c|c|c|c|}
\hline No. sutures & 4 & 5 & 6 & 7 & $P$ overall \\
\hline & $(n=16)$ & $(n=38)$ & $(n=27)$ & $(n=7)$ & \\
\hline Gender: & & & & & 0.287 \\
\hline - Male, n (\%) & $5(31.3 \%)$ & $10(26.3 \%)$ & $8(29.6 \%)$ & $5(71.4 \%)$ & \\
\hline - Female, n (\%) & $11(68.7 \%)$ & $28(73.7 \%)$ & $19(70.4 \%)$ & $2(28.6 \%)$ & \\
\hline Age, mean (SD), yrs & $50.0(14.6)$ & $44.4(9.56)$ & $45.6(12.6)$ & $48.5(20.9)$ & 0.656 \\
\hline $\mathrm{W}_{\mathrm{o}}$, mean $(\mathrm{SD}), \mathrm{kg}$ & $101.4(16.9)$ & $108.1(12.0)$ & $118.6(21.2)$ & $116.8(25.4)$ & 0.064 \\
\hline $\mathrm{BMI}_{0}$, mean $(\mathrm{SD}), \mathrm{kg} / \mathrm{m}^{2}$ & $36.9(3.96)$ & $38.6(3.38)$ & $41.2(5.31)$ & $42.3(7.44)$ & 0.052 \\
\hline N. of stitches, mean (SD) & $23.9(5.78)$ & $27.2(7.76)$ & $28.1(4.15)$ & $34.2(1.50)$ & 0.052 \\
\hline N. of stitches: & & & & & 0.001 \\
\hline - $<25, \mathrm{n}(\%)$ & $12(75.0 \%)$ & $17(44.7 \%)$ & $8(29.6 \%)$ & $0(0.00 \%)$ & \\
\hline - $25-30, n(\%)$ & $1(6.2 \%)$ & $12(31.6 \%)$ & $17(63.0 \%)$ & $0(0.00 \%)$ & \\
\hline - >30, n (\%) & $3(18.8 \%)$ & $9(23.7 \%)$ & $2(7.4 \%)$ & $7(100 \%)$ & \\
\hline $\mathrm{W}_{12}$, mean $(\mathrm{SD}), \mathrm{kg}$ & $84.7(15.0)$ & $89.2(12.3)$ & $97.4(20.9)$ & $96.8(23.9)$ & 0.217 \\
\hline $\mathrm{BMI}_{12}$, mean $(\mathrm{SD}), \mathrm{kg} / \mathrm{m}^{2}$ & $30.5(5.04)$ & $32.0(3.65)$ & $35.2(5.66)$ & $35.1(7.42)$ & 0.228 \\
\hline $\mathrm{WL}_{12}$, mean (SD), $\mathrm{kg}$ & $16.7(3.76)$ & $18.9(6.46)$ & $21.2(7.09)$ & $20.0(6.88)$ & 0.334 \\
\hline$\% \mathrm{EWL}_{12}$, mean (SD), \% & $55.6(17.1)$ & $50.5(18.5)$ & $42.8(19.1)$ & $40.0(31.4)$ & 0.296 \\
\hline $\mathrm{EWL}_{12}$ & & & & & 0.361 \\
\hline - $<25 \%, n(\%)$ & $0(0.00 \%)$ & $3(7.9 \%)$ & $2(7.4 \%)$ & $0(0.00 \%)$ & \\
\hline . >25\%, n (\%) & $16(100 \%)$ & $35(92.1 \%)$ & $25(92.6 \%)$ & $7(100 \%)$ & \\
\hline$\% \mathrm{TBWL}_{12}$, mean (SD), \% & $16.6(3.22)$ & $17.7(6.03)$ & $18.0(6.33)$ & $17.5(7.10)$ & 0.949 \\
\hline TBWL $_{12}:$ & & & & & 0.725 \\
\hline - < $<10 \%, n(\%)$ & $0(0.00 \%)$ & $3(7.9 \%)$ & $1(3.7 \%)$ & $0(0.00 \%)$ & \\
\hline . > $>10 \%, n(\%)$ & $16(100 \%)$ & $35(92.1 \%)$ & $26(96.3 \%)$ & $7(100 \%)$ & \\
\hline
\end{tabular}

W, weight; BMI, body mass index; WL, weight loss; EWL (\%), \% of excess weight loss; EWL >25\%, number of patients with EWL > 25\%; TBWL (\%), \% of total body weight loss; TBWL $>10 \%$ : number of patients with TBWL $>10 \%$. Data expressed at ${ }_{\mathrm{o}}$ : basal; ${ }_{12}$ : at 12 months.

was slightly lower than in our study. Another variant used a triangular pattern (mean of 6 sutures) with a second layer of sutures (average of 3 sutures) placed over the length of the central sleeve to further reduce gastric volume and reinforce the sleeve [15] ( Fig. 1). This strategy results in a more aggressive and expensive procedure, but it also does not improve results. It must be compared in the long term, at least 24 months.

In 2018 a new running "Z" suture stitch pattern ( $\triangleright$ Fig. 1) with four longitudinal parallel suture rows was reported [16]. The authors noted that a higher number of stitches $(>40)$ and homogenous distribution of force provided less suture tension. In addition, using only four sutures could favor a cheaper procedure. In our Lp procedures, we followed this pattern. Twelvemonth \%TBWL was slightly lower compared to our study, but with higher \%EWL, probably due to higher baseline mean BMI in our patients. In our series, this pattern seems to be the most effective, showing significant differences in \%EWL compared to other two patterns. This result may be somewhat mis- leading, because patients in the Lp group had lower basal mean BMI $(P=0.008)$, so these differences were not statistically seen in \%TBWL or WL $(\mathrm{kg})$. Another study [17] compared a unique combination of longitudinal compression sutures and " $U$ "shaped sutures. After 6 months, \%TBWL was greater in compression group compared to non-compression group.

Our first cases (years 2014-2015) were performed with the TBp pattern and coincided with the beginning of our learning curve and the goal of achieving a fully sutured gastric body (including fundus). These were probably the causes of using more sutures with the TBp pattern. In this sense, a large study [15] noted that more than 34 cases were predictive of weight loss. Experienced endoscopists are expected to achieve a reduction in length of procedure and number of plications, with progress plateauing at seven and nine cases, respectively [18]. The least number of sutures was used with the TMp pattern during period with the most experienced endoscopist (2018-2019) and in addition to the experience of leaving the fundus free of sutures. 
Table 4 Baseline and 12-month anthropometric data according to number of stitches

\begin{tabular}{|c|c|c|c|c|}
\hline N. of bites & $<25$ & $25-30$ & $>30$ & $P$ overall \\
\hline & $(n=37)$ & $(n=30)$ & $(n=21)$ & \\
\hline Gender: & & & & 0.168 \\
\hline - Male, n (\%) & $16(43.2 \%)$ & $10(33.3 \%)$ & $6(28.6 \%)$ & \\
\hline - Female, n (\%) & $21(56.8 \%)$ & $20(66.7 \%)$ & $15(71.4 \%)$ & \\
\hline Age, mean (SD), years & $42.5(9.94)$ & $48.3(12.9)$ & $49.2(14.3)$ & 0.189 \\
\hline $\mathrm{W}_{0}$, mean $(\mathrm{SD}), \mathrm{kg}$ & $119.1(19.0)$ & $105.1(10.4)$ & $104.0(19.6)$ & 0.010 \\
\hline $\mathrm{BMI}_{0}$, mean $(\mathrm{SD}), \mathrm{kg} / \mathrm{m}^{2}$ & $40.7(4.35)$ & $38.8(3.95)$ & $37.8(5.85)$ & 0.167 \\
\hline No. sutures, mean (SD) & $4.91(0.73)$ & $5.53(0.61)$ & $5.54(1.13)$ & 0.026 \\
\hline No. sutures: & & & & 0.001 \\
\hline - $4, \mathrm{n}(\%)$ & $12(32.4 \%)$ & $1(3.3 \%)$ & $3(14.3 \%)$ & \\
\hline - $5, \mathrm{n}(\%)$ & $17(46.0 \%)$ & $12(40.0 \%)$ & $9(42.9 \%)$ & \\
\hline - 6, n (\%) & $8(21.6 \%)$ & $17(56.7 \%)$ & $2(9.5 \%)$ & \\
\hline . 7, n (\%) & $0(0.00 \%)$ & $0(0.00 \%)$ & $7(33.3 \%)$ & \\
\hline $\mathrm{W}_{12}$, mean (SD), kg & $99.0(17.3)$ & $86.2(12.1)$ & $85.9(18.3)$ & 0.017 \\
\hline $\mathrm{BMI}_{12}$, mean $(\mathrm{SD}), \mathrm{kg} / \mathrm{m}^{2}$ & $36.1(5.10)$ & $32.8(4.46)$ & $31.7(5.62)$ & 0.151 \\
\hline $\mathrm{WL}_{12}$, mean (SD), $\mathrm{kg}$ & $20.1(5.82)$ & $19.0(7.07)$ & $18.4(6.47)$ & 0.726 \\
\hline$\% \mathrm{EWL}_{12}$, mean (SD), \% & $44.3(16.4)$ & $45.0(21.8)$ & $55.9(24.5)$ & 0.223 \\
\hline EWL12: & & & & 0.748 \\
\hline - $\leq 25 \%, n(\%)$ & $2(5.4 \%)$ & $2(6.7 \%)$ & $1(4.8 \%)$ & \\
\hline - >25\%, n (\%) & $35(94.6 \%)$ & $28(93.3 \%)$ & $20(95.2 \%)$ & \\
\hline$\% \mathrm{TBWL}_{12}$, mean (SD), \% & $17.2(4.85)$ & $17.9(6.37)$ & $17.8(6.32)$ & 0.911 \\
\hline $\operatorname{TBWL}_{12}:$ & & & & 1.000 \\
\hline - $<10 \%, \mathrm{n}(\%)$ & $2(5.4 \%)$ & $2(6.7 \%)$ & $0(0.0 \%)$ & \\
\hline . $>10 \%, n(\%)$ & $35(94.6 \%)$ & $28(93.3 \%)$ & $21(100 \%)$ & \\
\hline
\end{tabular}

W, weight; BMI, body mass index; WL, weight loss; EWL (\%), \% of excess weight loss; EWL > 25\%, number of patients with EWL > $25 \%$; TBWL (\%), \% of total body weight loss; TBWL $>10 \%$, number of patients with TBWL $>10 \%$. Data expressed at ${ }_{0}$ : basal; ${ }_{12}$ : at 12 months.

From the perspective of a cost-effectiveness ratio, a strategy with a lesser number of sutures should be prioritized. Although not demonstrated, the type of endoscope could influence the results. During our study, the OverStitch system was only adaptable to a double-channel endoscope. An adapter for singlechannel endoscopes has recently been designed.

Several studies demonstrate that ESG can reduce obesityassociated medical comorbidities. At 3 months, complete remission of AHT, T2DM, and DLP was observed in up to $100 \%$, $76 \%$, and $56 \%$ of patients, respectively $[14,15]$. Our results are not so encouraging, although $47.1 \%$ of patients with some initial comorbidity were discharged at 1 year, resolving $60 \%$ of total comorbidities. We analyzed only five major comorbidities and no other progressive diseases. In this sense, ESG has been proved to be an effective and safe alternative in patients with obesity and non-alcoholic fatty liver disease, stimulating weight loss and improving analytical and ultrasound parameters of liver fat and insulin-resistance [19].

In the three above-mentioned meta-analyses [4-6] the pooled incidence of serious AEs was only $1 \%$ to $2.2 \%$, mainly upper gastrointestinal bleeding and perigastric fluid collection. Surgery was rarely required and no mortality was reported. In our series, no major complications were reported. Only two patients with BTp pattern had somewhat longer pain/dysphagia without fever or bleeding, probably due to excessive gastric lumen restriction, without need for analytical or radiological study, self-limited at 48 hours, therefore, they were considered as minor incidences. All patients remained in grade I ClavienDindo complications classification, which includes any deviation from the normal postoperative course without need for extra pharmacological treatment or surgical, endoscopic, or radiological interventions [10]. 
When detailing durability and 12-month efficacy results between different bariatric endoscopic suturing pattern techniques, ESG appears to be superior to POSE [7, 20, 21], Endomina [22], EndoZip [23], GERDX [24] or RESTORe [25] devices. However, efficacy results at 6-month follow-up do not seem to be as good as with laparoscopic sleeve gastrectomy [26], but with more AEs and new-onset gastroesophageal reflux disorder than ESG.

Main limitations of our study were the retrospective design (even with prospective data collection), limited number of patients, confinement to two hospitals, follow-up limited to 12 months, patient inclusion from the beginning of the ESG learning curve in TBp, baseline BMI differences in pattern analyses, limited spread with respect to the number of sutures used, and absence of an endoscopic or radiographic surveillance control.

\section{Conclusion}

According to our results in limited 12-month follow-up, ESG provides good overall efficacy results: mean \%TBWL of $17.36 \%$ (TBWL $>10 \%$ in $95.5 \%$ patients), mean \%EWL of $46.41 \%$ (EWL> $25 \%$ in $94.3 \%$ patients) and resolution of $60 \%$ of major obesityassociated metabolic comorbidities. These results were not directly dependent upon suture pattern (although the Lp pattern appears to be related to a discreet higher \%EWL but with similar $\%$ TBWL), or the number of sutures or stitches applied. This demonstrates that the most important factor is to perform a complete and technically correct gastric tubular sleeve reduction, regardless of the formula, with adequate multidisciplinary follow-up. ESG appears to have a good safety profile with no major complications associated with any suture patterns used in this study.

\section{Acknowledgements}

The authors thank the Spanish Society for Gastrointestinal Endoscopy (SEED) for the fellowship granted in 2016 and the Teaching and Research Committee of Dexeus University Hospital of Barcelona in 2018. Both have contributed to and helped partially finance the study.

\section{Competing interests}

Dr. Espinet-Coll and Dr. Galvao-Neto are consultants and have received grants and personal fees from Apollo Endosurgery. Dr. GalvaoNeto has received grants and personal fees from Fractyl Labs, GI Dynamics, GI Windows, Olympus, Medtronic, and M.I. Tech.

\section{References}

[1] Hill C, Khashab MA, Kalloo AN et al. Endoluminal weight loss and metabolic therapies: current and future techniques. Ann N Y Acad Sci 2018; 1411: 36-52
[2] Kumar N, Sahdala HNP, Shaikh S et al. Endoscopic sleeve gastroplasty for primary therapy of obesity: initial human cases. Gastroenterology 2014; 146: S571-S2

[3] Abu Dayyeh BK, Rajan E, Gostout C]. Endoscopic sleeve gastroplasty: a potential endoscopic alternative to surgical sleeve gastrectomy for treatment of obesity. Gastrointest Endosc 2013; 78: 530-535

[4] Hedjoudje A, Dayyeh BA, Cheskin LJ et al. Efficacy and safety of endoscopic sleeve gastroplasty: a systematic review and meta-analysis. Clin Gastroenterol Hepatol 2020; 18: 1043-1053

[5] Singh S, Hourneaux de Moura DT, Khan A et al. Safety and efficacy of endoscopic sleeve gastroplasty worldwide for treatment of obesity: a systematic review and meta-analysis. Surg Obes Relat Dis 2020; 16 : 340-351

[6] Li P, Ma B, Gong S et al. Efficacy and safety of endoscopic sleeve gastroplasty for obesity patients: a meta-analysis. Surg Endosc 2020; 34: $1253-1260$

[7] Gys B, Plaeke P, Lamme B et al. Endoscopic gastric plication for morbid obesity: a systematic review and meta-analysis of published data over time. Obes Surg 2019; 29: 3021-3029

[8] Espinet Coll E, López-Nava Breviere G, Nebreda Durán J et al. Spanish Consensus Document on Bariatric Endoscopy. Part 1. General considerations. Rev Esp Enferm Dig 2018; 110: 386-399

[9] Alberti KG, Eckel RH, Grundy SM et al. International Diabetes Federation Task Force on Epidemiology and Prevention. Hational Heart, Lung, and Blood Institute. World Heart Federation. American Heart Association. International Atherosclerosis Society. International Association for the Study of Obesity. Harmonizing the metabolic syndrome: a joint interim statement of the International Diabetes Federation Task Force on Epidemiology and Prevention; National Heart, Lung, and Blood Institute; American Heart Association; World Heart Federation; International Atherosclerosis Society; and International Association for the Study of Obesity. Circulation 2009; 120: 16401645

[10] Clavien PA, Barkun ], de Oliveira ML et al. The Clavien-Dindo classification of surgical complications: five-year experience. Ann Surg 2009; 250: 187-196

[11] Abu Dayyeh BK, Acosta A, Camilleri M et al. Endoscopic sleeve gastroplasty alters gastric physiology and induces loss of body weight in obese individuals. Clin Gastroenterol Hepatol 2017; 15: 36-43.e1

[12] Hajifathalian K, Ang B, Dawod QM et al. Long-term follow up and outcomes after endoscopic sleeve gastroplasty for treatment of obesity (5 year data). Gastrointest Endosc 2019; 6: AB58

[13] Lopez-Nava G, Sharaiha RZ, Vargas EJ et al. Endoscopic sleeve gastroplasty for obesity: a multicenter study of 248 patients with 24 months follow-up. Obes Surg 2017; 27: 2649-2655

[14] Alqahtani A, Al-Darwish A, Mahmoud AE et al. Short-term outcomes of endoscopic sleeve gastroplasty in 1000 consecutive patients. Gastrointest Endosc 2019; 89: 1132-1138

[15] Sharaiha RZ, Kumta NA, Saumoy M et al. Endoscopic sleeve gastroplasty significantly reduces body mass index and metabolic complications in obese patients. Clin Gastroenterol Hepatol 2017; 15: $504-$ 510

[16] Graus Morales ], Crespo Pérez L, Marques A et al. Modified endoscopic gastroplasty for the treatment of obesity. Surg Endosc 2018; 32: 3936-3942

[17] Glaysher MA, Moekotte AL, Kelly J. Endoscopic sleeve gastroplasty: a modified technique with greater curvature compression sutures. Endosc Int Open 2019; 7: E1303-E1309

[18] Hill CH, El Zein M, Agnihotri A et al. Endoscopic sleeve gastroplasty: the learning curve. Endosc Int Open 2017; 5: E900-E904

[19] Espinet Coll E, Vila Lolo C, Díaz Galán P et al. Bariatric and metabolic endoscopy in the handling of fatty liver disease. A new emerging approach? Rev Esp Enferm Dig 2019; 111: 283-293 
[20] Sullivan S, Swain JM, Woodman G et al. Randomized sham-controlled trial evaluating efficacy and safety of endoscopic gastric plication for primary obesity: The ESSENTIAL trial. Obesity (Silver Spring) 2017; 25: 294-301

[21] Khan Z, Khan MA, Hajifathalian K et al. Efficacy of endoscopic interventions for the management of obesity: a meta-analysis to compare endoscopic sleeve gastroplasty, aspire assist, and primary obesity surgery endolumenal. Obes Surg 2019; 29: 2287-2298

[22] Huberty V, Machytka E, Boškoski I et al. Endoscopic gastric reduction with an endoluminal suturing device: a multicenter prospective trial with 1-year follow-up. Endoscopy 2018; 50: 1156-1162
[23] Lopez-Nava G, Asokkumar R, Rull A et al. Safety and feasibility of a novel endoscopic suturing device (EndoZip TM) for treatment of obesity: first-in-human study. Obes Surg 2020; 30: 1696-1703

[24] Wannhoff A, Hofmann C, Meier B et al. Endoscopic sleeve gastroplasty for severe obesity by full-thickness suturing using the GERDX device. Endoscopy 2019; 51: E34-E35

[25] Brethauer SA, Chand B, Schauer PR et al. Transoral gastric volume reduction as intervention for weight management: 12-month follow-up of TRIM trial. Surg Obes Relat Dis 2012; 8: 296-303

[26] Fayad L, Adam A, Schweitzer M et al. Endoscopic sleeve gastroplasty versus laparoscopic sleeve gastrectomy: a case-matched study. Gastrointest Endosc 2019; 89: 782-788 